\title{
ENERGY LOSS OF PROTON AND LEAD BEAMS IN THE CERN-SPS
}

\author{
J. Arnold, T. Bohl, H. Burkhardt, R. Cornali, K. Cornelis, G. Engelmann, \\ R. Giachino, A. Hofmann, M. Jonker, T. Linnecar, M. Meddahi, L. Normann, \\ E. Shaposhnikova, A. Wagner and B. Zotter, CERN, 1211 Geneva 23, Switzerland
}

\section{Abstract}

The energy loss of an unbunched beam circulating in the CERN-SPS has been obtained from the observed frequency change of a longitudinal Schottky signal. This experiment was carried out for protons at 14, 120 and $270 \mathrm{GeV} / \mathrm{c}$ and for lead ions $\mathrm{Pb}_{208}^{82}$ at $Z \cdot 270 \mathrm{GeV} / \mathrm{c}$ momentum. The dominant effects which determine the energy loss are synchrotron radiation, ionization of the residual gas and parasitic mode loss in the resistive longitudinal impedance. Since all the protons in a lead nucleus radiate coherently the synchrotron radiation is proportional to $\mathrm{Z}^{2}$ like the other effects. The experimental results are analyzed and the contributions of the individual effects determined. Using an impedance of $|Z / n| \approx 12 \Omega$ gives the best fit through the experimental data.

\section{INTRODUCTION}

This experiment was carried out to measure the energy loss due to the synchrotron radiation emitted by protons and lead ions and due to the resistive wall impedance but also the energy loss due to ionization of the rest gas had to be considered.

The synchrotron radiation of a radially accelerated charge in free space is well known. For the fully stripped lead ions $P b_{208}^{82}$ it has to be considered that the charge is $q=Z e$ and all the 82 protons radiate coherently resulting in a emitted power being proportional to $Z^{2}$. Furthermore the shielding effect by the vacuum chamber has to be taken into account. For the energy loss caused by the impedance of the beam surroundings we have to consider that the unbunched beam consists of point charges without any phase relation between them. Each charge produces an electric field concentrated in the transverse direction with an opening angle of about $\pm 1 / \gamma$ which induces on the wall a short current pulse of length proportional to $1 / \gamma$. By measuring the loss for different beam energies some information about the resistive wall impedance can be obtained. In the following we will discuss the different effects leading to an energy loss and calculate the expected magnitude for the conditions used in the experiment. We make some estimates of the impedance based on earlier measurements done in the SPS and similar machines and we will use the actual measured pressures of the residual gas to estimate the loss due to ionization. The expected effects are presented and compared with the experimental results.

\begin{tabular}{|c|c|c|c|}
\hline circumference & $2 \pi R$ & $\mathrm{~m}$ & 6912 \\
bending radius & $\rho$ & $\mathrm{m}$ & 741.3 \\
revolution freq. & $\omega_{0}$ & $\mathrm{~s}^{-1}$ & $2 \pi 43400$ \\
mom. compaction & $\alpha_{c}$ & & 0.0018 \\
chamber height & $h_{\mathrm{MBA}}$ & $\mathrm{mm}$ & 24 \\
", & $h_{\mathrm{MBB}}$ & $\mathrm{mm}$ & 41 \\
eff. chamber radius & $b$ & $\mathrm{~mm}$ & 35 \\
\hline
\end{tabular}

Table 1: Relevant SPS parameters

\section{KINEMATICS}

A particle with charge $q$, rest mass $m_{0}$ and momentum $p$ circulates in a transverse magnetic field $B$ on an orbit with radius of curvature $\rho$ given by

$$
\frac{1}{\rho}=\frac{q B}{p}=\frac{q B}{m_{0} c \beta \gamma} .
$$

Since the bending radius and the field are the same for protons $\left(q=e, m_{0}=m_{p}\right)$ and lead ions $(q=Z e$, $\left.m_{0}=m_{P b}\right)$ we have the relation

$$
p_{P b}=m_{P b} c \beta_{P b} \gamma_{P b}=Z p_{p}=Z m_{p} c \beta_{p} \gamma_{p}
$$

As approximations we take for the mass of the lead isotope $\mathrm{Pb}_{208}^{82}$ ion $m_{P b}=A m_{p}$ and set $\beta=1$ except where it is subtracted from a quantity close to unity. With this we get for the two particles circulating on the same orbit in the same magnetic field

$$
\frac{\gamma_{P b}}{\gamma_{p}} \approx \frac{Z}{A}=\frac{82}{208}=0.394
$$

In the experiment the energy loss has been determined by observing the change of the frequency $\omega_{s}=2 \pi f_{s}=k \omega_{0}$ of a Schottky signal being a harmonic of the revolution frequency $\omega_{0}$ which depends on the momentum

$$
\frac{d \omega_{0}}{\omega_{0}}=-\eta \frac{d p}{p} \approx-\eta \frac{d E}{E} \text { with } \eta=\alpha_{c}-\frac{1}{\gamma^{2}}
$$

where $\alpha_{c}$ is the momentum compaction. The relative energy loss per unit time or the energy loss $U$ per turn is obtained from the observed time variation of the Schottky signal frequency

$$
\frac{1}{E} \frac{d E}{d t}=-\frac{1}{\eta f_{S}} \frac{d f_{S}}{d t} \text { and } U=\frac{E}{\eta f_{0} f_{S}} \frac{d f_{S}}{d t} .
$$

Table 1 lists the relevant parameters of the SPS. 


\section{SYNCHROTRON RADIATION}

For the calculation of the energy loss due to synchrotron radiation we use the equation

$$
U_{s}=\frac{q^{2} \beta^{3} \gamma^{4}}{3 \epsilon_{0} \rho} \approx \frac{q^{2} \gamma^{4}}{3 \epsilon_{0} \rho} .
$$

Here $q$ is the charge of the circulating particle. For the proton it is just the elementary charge $q=e$. However, for the lead ion we have $q=Z e$ since the nucleus containing $Z$ protons can be considered as a point charge within the scale of the wavelength of the emitted radiation, in other words, all the protons of the lead nucleus radiate coherently. Using the ratio between the Lorentz factors for protons and the lead ions (3) we get for the energy loss ratio

$$
\frac{U_{P b}}{U_{P}}=\left(\frac{\gamma_{P b}}{\gamma_{P}}\right)^{4} Z_{P b}^{2}=\frac{Z_{P b}^{6}}{A_{P b}^{4}}=\frac{82^{6}}{208^{4}}=162.4 .
$$

The relative energy loss per unit time

$$
-\frac{1}{E} \frac{d E}{d t}=f_{0} \frac{U_{s}}{E}=\frac{e^{2} Z^{2} f_{0} \gamma^{3}}{3 \epsilon_{0} A m_{P} c^{2} \rho}=\frac{1}{2} \frac{1}{\tau_{\mathrm{rad}}},
$$

is half the total radiation damping rate $1 / \tau_{\text {rad }}$ and has the ratio

$$
\frac{\left(\frac{1}{E} \frac{d E}{d t}\right)_{P b}}{\left(\frac{1}{E} \frac{d E}{d t}\right)_{P}}=\frac{Z^{5}}{A^{4}}=1.98 .
$$

We arrive at this somewhat surprising result that the lead ions have a larger damping rate and move faster to the inside than the protons. But this is true only in free space and the radiation from lead, being at lower frequencies, is reduced by the vacuum chamber more than the one from protons. The spectrum of the emitted radiation is characterized by the critical frequency $\omega_{c}$ of wavelength $\lambda_{c}$ which divides it into two parts of equal power

$$
\omega_{c r i t}=\frac{3 c \gamma^{3}}{2 \rho}, \quad \lambda_{c}=\frac{4 \pi \rho}{\gamma^{3}} .
$$

So far the synchrotron radiation has been calculated for the case of free space. The presence of the conducting vacuum chamber may reduce the emitted radiation because the lower frequencies cannot propagate. The calculation of this suppression is quite complicated. We use here an equation from $[1,2]$ which gives the frequency $\omega_{t h}$ above which radiation is possible

$$
\frac{\omega_{t h}}{\omega_{0}}=\sqrt{\frac{2}{3}}\left(\frac{\pi \rho}{h}\right)^{3 / 2} .
$$

Here, $h$ is the full height of the chamber listed in table 1 . The emitted power is calculated for the two types of magnet chambers using the simplifying assumption that the synchrotron radiation spectrum lying above $\omega_{t h}$ is not changed while the part below is completely suppressed.

\section{IONIZATION OF THE RESIDUAL GAS}

The energy loss due to ionization of the residual is [3]

$$
\begin{gathered}
\frac{d E}{d X}=-K \frac{Z^{2}}{\beta^{2}} \frac{Z^{*}}{A^{*}}\left[\frac{1}{2} \ln \left(\frac{2 m_{e} c^{2} \beta^{2} \gamma^{2} T}{I^{2}}\right)-\beta^{2}-\frac{\delta}{2}\right] \\
\text { with } T=\frac{2 m_{e} c^{2} \beta^{2} \gamma^{2}}{1+2 \gamma m_{e} / M+\left(m_{e} / M\right)^{2}}
\end{gathered}
$$

Here, $X$ is the mass per unit cross section (usually given in $\left.\left[\mathrm{gr} / \mathrm{cm}^{2}\right]\right), A^{*}$ and $Z^{*}$ are atomic weight and number of the residual gas, $K=4 \pi N_{A} r_{e} m_{e} c^{2}=0.307 \mathrm{~g}^{-1} \mathrm{~cm}^{2}$ and $M$ the mass of the nucleus. For our application we can neglect the density effect $\delta / 2$ and set $\beta=1$. The quantity $I$ is the mean excitation energy which is about $19.2 \mathrm{eV}$ for $\mathrm{H}_{2}, 82$ $\mathrm{eV}$ for $\mathrm{N}_{2}$ and $95.1 \mathrm{eV}$ for $\mathrm{O}_{2}$, [3].

Based on earlier measurements the relative partial pressures of the residual gas were estimated to be about $52 \%$ $\mathrm{H}_{2}, 30 \% \mathrm{H}_{2} \mathrm{O}$ and $18 \% \mathrm{~N}_{2}$. For our calculation we split the water molecule and get for the partitions of the partial densities $11 \%$ for $\mathrm{H}, 66 \%$ for $\mathrm{N}$ and $23 \%$ for $\mathrm{O}$. The pressure has been measured with about 70 gauges around the ring and averaged taking the distance between the measurement points and the pressure bumps in between into account. These values are listed in table 2. For three of the experiments a lower pressure was obtained by using the sublimation pumps.

\section{PARASITIC MODE LOSS}

The resistive impedance of the vacuum chamber leads to an energy loss of the particles in the coasting (unbunched) beam. Each charge produces an electric field which is by the relativistic contraction concentrated in the transverse direction with a typical opening angle of $\pm 1 / \gamma$. It induces on the conducting wall surface a current pulse of rms length [4] $\sigma_{t}=b /(\sqrt{2} c \gamma)$ where $b$ is the radius of a circular cylinder which approximates the different cross sections of the vacuum chamber. The energy loss is proportional to an integral over the product of resistive impedance times the Fourier transformation of wall pulse. By measuring this loss for different energies and hence, for different pulse lengths $\sigma_{t}$, we get some information about the impedance. At high frequencies the impedance is mainly due to diffraction on the aperture changes which decreases like $1 / \sqrt{\omega}$ and, to a lesser extent, due to the smooth resistive wall which increases like $\sqrt{\omega}$. For the diffraction contribution we assume that the impedance has a maximum around 1.3 $\mathrm{GHz}$ similar to the PS and ISR rings. Based on various SPS measurements we take for the characteristic impedance at low frequencies $|Z / n|=10 \Omega$. The resistive wall effect is calculated for a stainless steel chamber with radius $b$. The estimated parasitic mode loss factor $k_{p m}$ are listed in table 2 for both contributions. This quantity is related to the energy loss by $U=q^{2} k_{p m}$. 


\begin{tabular}{|c|c|c|c|c|c|c|c|c|}
\hline parameters & $\begin{array}{l}\text { particle } \\
p / Z \\
\gamma \\
\eta \\
\end{array}$ & $\mathrm{GeV} / \mathrm{c}$ & $\begin{array}{c}\text { proton } \\
14 \\
14.96 \\
-0.00267\end{array}$ & \multicolumn{2}{|c|}{$\begin{array}{c}\text { proton } \\
120 \\
127.9 \\
0.00174\end{array}$} & \multicolumn{2}{|c|}{$\begin{array}{c}\text { proton } \\
270 \\
287.8 \\
0.00179\end{array}$} & $\begin{array}{c}\text { lead } \\
270 \\
113.4 \\
0.00172 \\
\end{array}$ \\
\hline $\begin{array}{l}\text { Synch. rad. } \\
\text { (free space) } \\
\text { reduced }\end{array}$ & $\begin{array}{l}\lambda_{\text {crit. }} \\
U_{s} / Z^{2} \\
U_{s} / Z^{2}\end{array}$ & $\begin{array}{l}\mathrm{mm} \\
\mathrm{m} \mathrm{eV} \\
\mathrm{m} \mathrm{eV}\end{array}$ & $\begin{array}{l}94 \\
0.0 \\
0.0\end{array}$ & \multicolumn{2}{|c|}{$\begin{array}{l}1.5 \\
2.2 \\
0.3\end{array}$} & \multicolumn{2}{|c|}{$\begin{array}{l}0.13 \\
55.8 \\
50.3\end{array}$} & $\begin{array}{l}2.1 \\
1.3 \\
0.1\end{array}$ \\
\hline Impedance & $\begin{array}{l}\sigma \\
k_{p m} / \text {-diff. } \\
k_{p m} \text {-res. w. } \\
U_{p m} / Z^{2}\end{array}$ & $\begin{array}{l}\mathrm{mm} \\
\mathrm{V} / \mathrm{pC} \\
\mathrm{V} / \mathrm{pC} \\
\mathrm{m} \mathrm{eV}\end{array}$ & $\begin{array}{c}1.65 \\
3560 \\
40 \\
0.6\end{array}$ & \multicolumn{2}{|c|}{$\begin{array}{c}0.194 \\
10470 \\
940 \\
1.8\end{array}$} & \multicolumn{2}{|c|}{$\begin{array}{c}0.086 \\
15610 \\
3200 \\
3.0\end{array}$} & $\begin{array}{c}0.217 \\
9826 \\
800 \\
1.7\end{array}$ \\
\hline Ionization & $\begin{array}{l}P \\
\text { density } \\
U_{i} / Z^{2}\end{array}$ & $\begin{array}{l}\text { nTorr } \\
\mu \mathrm{g} / \mathrm{m}^{3} \\
\mathrm{meV}\end{array}$ & $\begin{array}{c}4.6 \\
0.031 \\
5.1\end{array}$ & $\begin{array}{c}9.35 \\
0.063 \\
13.7\end{array}$ & $\begin{array}{c}4.6 \\
0.031 \\
6.8\end{array}$ & $\begin{array}{c}9.35 \\
0.063 \\
14.9 \\
\end{array}$ & $\begin{array}{c}4.6 \\
0.031 \\
7.3\end{array}$ & $\begin{array}{c}7.7 \\
0.052 \\
12.1\end{array}$ \\
\hline Total estimated & $\begin{array}{l}U \\
U-U_{s}\end{array}$ & $\begin{array}{l}\mathrm{meV} \\
\mathrm{m} \mathrm{eV}\end{array}$ & $\begin{array}{l}5.7 \\
5.7\end{array}$ & $\begin{array}{l}15.8 \\
15.5\end{array}$ & $\begin{array}{l}8.9 \\
8.6\end{array}$ & $\begin{array}{l}68.2 \\
17.9\end{array}$ & $\begin{array}{l}60.6 \\
10.3\end{array}$ & $\begin{array}{l}13.9 \\
13.8\end{array}$ \\
\hline Measured & $\begin{array}{l}U / Z^{2} \\
\left(U-U_{s}\right) / Z^{2}\end{array}$ & $\begin{array}{l}\mathrm{meV} \\
\mathrm{m} \mathrm{eV}\end{array}$ & $\begin{array}{l}9.7 \\
9.1\end{array}$ & $\begin{array}{l}20.7 \\
20.4 \\
\end{array}$ & $\begin{array}{l}16.3 \\
16.0 \\
\end{array}$ & $\begin{array}{l}74.7 \\
20.4\end{array}$ & $\begin{array}{l}62.5 \\
12.2 \\
\end{array}$ & $\begin{array}{l}16.3 \\
16.2 \\
\end{array}$ \\
\hline
\end{tabular}

Table 2: Top:Estimated energy losses of protons and $\mathrm{Pb}_{208}^{82}$ due to synchrotron radiation, due to parasitic modes (using $|Z / n|=10 \Omega$ ) and due to ionization (taking for the partial pressures $52 \% \mathrm{H}_{2}, 30 \% \mathrm{H}_{2} \mathrm{O}$ and $18 \% \mathrm{~N}_{2}$ ). Bottom: Results of the measurements and their correction for synchrotron radiation.

\section{EXPERIMENTS AND RESULTS}

In the experiments a beam was accelerated to the desired energy where it was debunched and left coasting. A longitudinal Schottky signal around $\omega_{S}=2 \pi 10^{9} \mathrm{~s}^{-1}$ was observed and its central frequency measured as a function of time for about 3 hours. From this the energy loss $U$ per turn was obtained using (5). For the case of $14 \mathrm{GeV}$ protons the life time was only about an hour which made the measurement less accurate. All other cases had a long life time. The variation of the dipole magnet field was checked and found to be negligible. The results are listed at the lower part of table 2 .

The measured energy losses are corrected for synchrotron radiation $U-U_{s}$ and listed in table 2. In order to determine the contributions of the impedance and of the ionization to the energy loss we use the calculated values of the corresponding energy losses and calculate the factors by which they have to be multiplied in order to get the best agreement with the measurements. This procedure was carried out for all proton measurements and for the case which also included the lead measurements. The two approaches resulted in differences of about $30 \%$ for the impedance and $10 \%$ for the pressure. Taking both with the appropriate weight we find for the impedance and the ionization loss which give the best fit through the measured data

$$
\left|\frac{Z}{n}\right| \approx 12 \pm 4 \Omega \text { and } U_{i} \text { (meas.) } \approx 1.4 U_{i} \text { (exp.). }
$$

The result for the impedance is good agreement with other measurements done at the SPS [5]. The obtained ionization loss could be explained by a slightly higher pressure or a different gas composition. In fact a distribution of 52\%
$\mathrm{H}_{2}, 30 \% \mathrm{H}_{2} \mathrm{O}, 4 \% \mathrm{~N}_{2}, 10 \% \mathrm{CO}$ and $4 \%$ of other gases like Argon could be more realistic. In any case the measurements have errors large enough to explain differences with expectations.

\section{ACKNOWLEDGEMENTS}

We thank the LEP-SPS operation group for setting up the coasting beams for this experiments. We also thank U. Wehrle for his help to obtain the Schottky signal and H. Jacob for measuring the beam life time.

\section{REFERENCES}

[1] S. Heifets, A. Michailichenko: Poc. of the 1991 Part. Accel. Conference, p. 458.

[2] S.A. Kheifets, B. Zotter; CERN SL-95/92 (AP), 1995.

[3] Review of Part. Phy.; Phys. Rev. D, Vol 54, 1996.

[4] A. Hofmann, T. Risselada; IEEE-NS 30 (1983), p. 2400.

[5] L. Vos; CERN SPS/86-21(MS), 1996. 